\title{
Rapid Analysis as an Integral Part of Additive Manufacturing
}

J. Goulden ${ }^{1}$, P. Trimby ${ }^{1}$, J Porter ${ }^{2}$ and M Hiscock ${ }^{1}$

1. Oxford Instruments NanoAnalysis, Halifax Road, High Wycombe, UK.

2. UES Inc, Dayton, Ohio, USA.

Additive layer manufacturing (ALM) is a form of 3D printing where components are formed at or near to the final shape by the sequential addition of layers of material. ALM benefits from both reduced machining cost and reduced waste. It is used for forming complex components of expensive to machine materials, such as the titanium alloys used in aerospace applications. The reduced tooling and machining in ALM make the process significantly faster than conventional manufacturing.

Materials analysis is an integral part of the additive manufacturing process, from quality control of the starting powder through to assessment of the effects of post manufacturing heat treatments. This will be demonstrated here using Ti 64 alloys used in aerospace applications. These alloys are manufactured by 3D printing, and then processed by hot isostatic pressing (HIP) to reduce voids and likely failures. The Ti microstructures formed by ALM evolve across multiple length scales and are anisotropic, which can negatively impact the component's mechanical properties.

While the final microstructure is critical in the component's performance, the final part is only ever as good as the source powder [1]. As such the quality of the source powder is investigated through automated particle analysis, where in excess of 30,000 particles can be analysed per hour. This technique is used to identify chemical impurities in the source powder, and to ensure that these impurities do not exceed a certain level. This is illustrated in Figure 1.

The final Ti components are also analysed. The microstructure is measured using electron backscattered diffraction (EBSD). This will indicate the impact of both the 3D printing and HIP processing parameters on the final component. The microanalysis results can be correlated with the results of mechanical testing and in order to be used to indicate the performance of the components in service [2]. The analysis and subsequent data processing was undertaken using the Symmetry EBSD detector and the AZtec microanalysis software.

The samples were characterised over two length scales. High resolution data sets were collected to understand the relationship between the alpha and remnant beta titanium and to examine the characteristic basket weave structure. Figure 2 shows higher resolution maps from three different HIP treatments. With higher temperature HIP there is a clear grain coarsening and increase in beta Ti.

A lower resolution data set collected over a much larger area gave an overview of the macro structure of the sample, specifically the large scale original beta grain structure. This data set was collected using automated large area mapping, where data is collected from multiple fields and montaged together. Here an overnight data set was collected from 800 fields with in excess of 50 million data points (Figure $3)$.

This fast and detailed microanalysis enabled a thorough understanding of the sample and was correlated with mechanical properties, including strength and fatigue resistance. 
References:

[1] Y Sun et al., Additive Manufacturing 21 (2018), p. 544.

[2] AT Sidambe et al., International Journal of Refractory Metals and Hard Materials 78 (2019), p. 254.

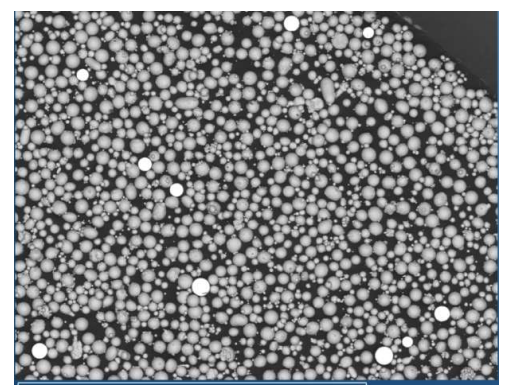

\section{Figure 1.}

(a)BSE Image showing particles in Titanium powder sample

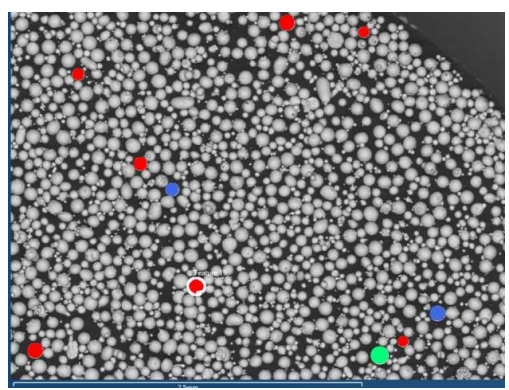

(b) Impurity Particles meeting detection criteria are shown in color

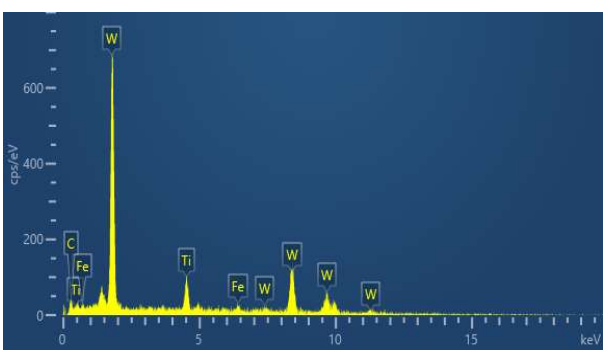

(c) Typical spectrum acquired from a marked $\mathrm{W}$ particle.
No HIP
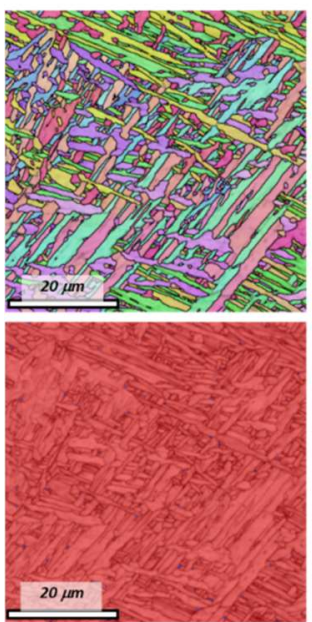

HIP 1
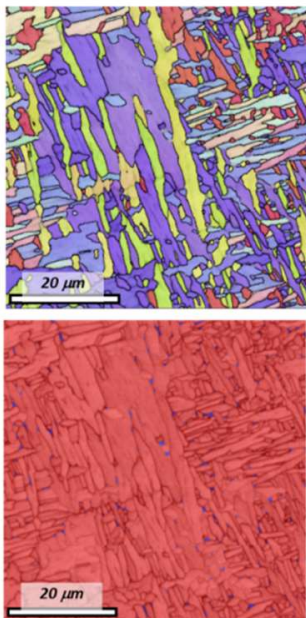

HIP 2

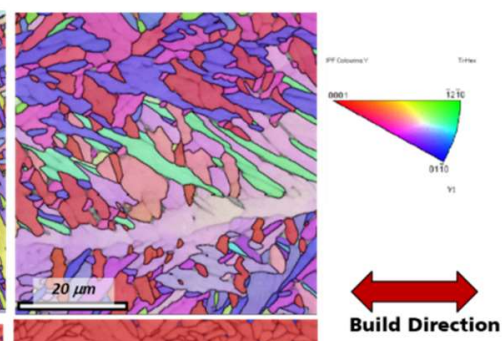

$\alpha-$ red

$\beta$-blue

Figure 2. High resolution maps from three different HIP treatments, higher temperature HIP treatment HIP 2 Temp > HIP 1 Temp) results in grain coarsening and increase in regrown $\beta \mathrm{Ti}$

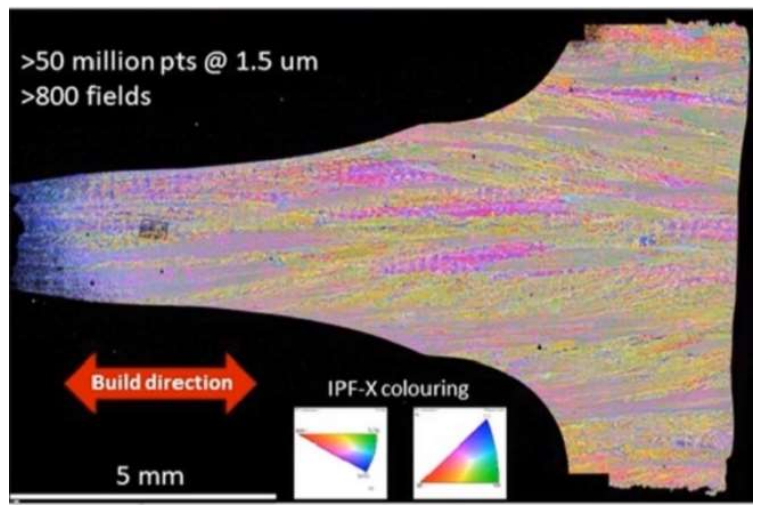

Figure 3. Montaged large area EBSD orientation map of a mechanical test sample. 